\title{
Markante Akzente - EQUAM wartet mit Innovationen auf
}

Kurt Hess

1 SAMW - Schweizerische Akademie der Medizinischen Wissenschaften: Zertifizierung im medizinischen Kontext Empfehlungen der SAMW; Juni 2011.

2 Indikatoren und Standards aller Module sind auf www.equam.ch unter Dienstleistungen einsehbar.

Korrespondenz:

Dr. med. et lic. oec. Kurt Hess

Sophienstrasse 2

CH-8032 Zürich

Tel. 0432684570

kurt.hess[at]hin.ch

Die Produkte von EQUAM sind seit der Gründung 1999 laufend ausgebaut worden. Seit langem sind die Indikatorensätze für Grundversorgerpraxen, Ärztenetze und die klinische Ergebnis- und Indikationsqualität etabliert. Nun stellt die Stiftung neue Schwerpunkte vor. So sind die vielbeachteten klinischen Module auf Anregung aus der Ärzteschaft als separate Zertifikate verfügbar. Neu wird auch ein Zertifikat für Spezialärzte angeboten. In Vorbereitung ist ferner eine französische Version der Zertifizierungsunterlagen.

Die medizinische Qualitätssicherung und die Patientensicherheit gehören zu den aktuellsten Initiativen im Gesundheitswesen. Die Entwicklung dazu wurde Anfang der Neunzigerjahre eingeleitet und insbesondere durch das KVG 1996 mit den - mehr oder weniger - klaren Postulaten zur Qualitätssicherung konkretisiert. Seither - und da sind bis dato über 16 Jahre vergangen - ist durch zahlreiche parlamentarische Vorstösse und einige politische Entscheide mit wechselndem Erfolg auf die Umsetzung hingearbeitet worden. Abgeschlossen ist dieser Prozess aber beileibe nicht.

EQUAM (Externe Qualitätssicherung in der Medizin) ist in den ersten Jahren dieser Entwicklung gegründet worden und hat sie in den bald 14 Jahren ihres Bestehens wesentlich mitgeprägt. Dabei hat sich die Stiftung sukzessive zu einer professionellen Institution entwickelt, die als einzige der Qualitätssysteme für die ambulante Versorgung die Empfehlungen der SAMW $^{1}$ erfüllt. Die Indikatorensätze ${ }^{2}$, von Ärzten für Ärzte erarbeitet und wissenschaftlich validiert, sind modular aufgebaut (Tab. 1). Die Zertifikate für Arztpraxen (Modul A), für Ärztenetze (Modul B) sowie für

Tabelle 1

Modularer Aufbau des EQUAM Instrumentariums.

EQUAM Indikatoren und Standards

Modul A für Arztpraxen

Basis EPA (Europäisches Praxisassessment)

Modul B für Ärztenetze

(Zusatzmodul nach Zertifikation der Netzärzte)

Module C der Ergebnis- und Indikationsqualität

C1: Arterielle Hypertonie

C2: Diabetes mellitus Typ 2

C3: Koronare Herzkrankheit

Erhebung der Patientenzufriedenheit Basis EUROPEP

\section{Qualitätszertifikat}

«Zertifizierte Praxisqualität (EPA)»

«Zertifizierte Netzqualität

(Integrierte Versorgung)»

«Zertifizierte Behandlungsqualität» Neu: Separates Angebot

(bisher als Zusatzmodul nach Zertifikation nach Modul A)

Patientenbefragung für Hausarztpraxen, Pädiater und Spezialarztpraxen die klinische Behandlung nach Ergebnis- und Indikationsqualität (Module C) sind inzwischen gut eingeführt. EQUAM ist für alle drei Module beim Bund (seco) als Zertifizierer akkreditiert.

\section{Separates Zertifikat zur Ergebnis- und Indikationsqualität}

Die Entwicklung im klinischen Qualitätsmanagement hat schon früh auf die zentrale Bedeutung der Ergebnis- und Indikationsqualität hingewiesen. Dies hat den Stiftungsrat 2005 bewogen, ein Projektteam mit der Entwicklung diagnosespezifischer Indikatoren dieser klinischen Qualitätsdimensionen zu beauftragen. Bis dato sind drei dieser Module erarbeitet und erfolgreich akkreditiert worden, weitere sind in Vorbereitung (Tab. 2).

Tabelle 2

Die klinischen Module C (Ergebnis- und Indikationsqualität).

\begin{tabular}{|c|c|}
\hline 2005 & $\begin{array}{l}\text { Auftrag Stiftungsrat zum Projekt Klinische } \\
\text { Indikatoren der Ergebnis- und Indikations- } \\
\text { qualität }\end{array}$ \\
\hline 2006-07 & $\begin{array}{l}\text { Pilotmodul C1: Arterielle Hypertonie } \\
\text { (Koop. AOK/AQUA) }\end{array}$ \\
\hline 2008 & $\begin{array}{l}\text { Erweiterung des Geltungsbereichs der } \\
\text { Akkreditierung auf das Modul C } \\
\text { (klinische Indikatoren und Standards) }\end{array}$ \\
\hline 2008 & Förderpreis des Forums Managed Care \\
\hline 2008-09 & $\begin{array}{l}\text { Modul C2: Diabetes mellitus } \\
\text { (Koop. AQUA/AOK, mediX Zürich) }\end{array}$ \\
\hline 2009 & $\begin{array}{l}\text { Modul C3: Koronare Herzkrankheit } \\
\text { (Koop. EPA/Swisspep) }\end{array}$ \\
\hline 2011 & $\begin{array}{l}\text { Modul C4: Arbeitsfähigkeits-Assessment } \\
\text { SUVA (noch kein formelles Zertifikat) }\end{array}$ \\
\hline seit 2011 & $\begin{array}{l}\text { in Vorbereitung: } \\
\text { Modul C5: Medikationssicherheit }\end{array}$ \\
\hline
\end{tabular}

Die Module C sind zunächst als reine Zusatzmodule konzipiert worden. Eine solche Zertifizierung setzte voraus, dass zuvor das Modul A für Arztpraxen mit dem entsprechenden Zertifikat erfolgreich abgeschlossen worden ist. Diese Bedingung wurde von manchen Ärzten als Hemmnis empfunden, sich einem Nachweis der klinischen Behandlungsqualität zu unterziehen. Der Stiftungsratsausschuss hat deshalb entschieden, die Module $\mathrm{C}$ auch separat anzubieten, dies unter dem Titel «Zertifizierte Behandlungsqualität». Die Fachgruppe Indikatoren und Standards hat zudem kürzlich die drei bis dahin verfügbaren klinischen Module von Grund auf überarbeitet und aktualisiert. 


\section{Basis-Zertifikat für Spezialärzte}

Eine zweite Erweiterung der Produktepalette ist zusammen mit unserem Kooperationspartner AQUA ${ }^{3}$ bereitgestellt worden. Während die bisherigen Zertifikate wie auch das EPA-System die Hausarztmedizin fokussieren, ist von einzelnen Spezialisten angeregt worden, für sie ein eigenes Label zu entwickeln. Die entsprechenden Indikatoren basieren erneut auf dem EPA-System, enthalten aber neu eine Zuweiserbefragung. Bei der Version für die Pädiatrie wird die Patientenzufriedenheit über eine Befragung der Eltern und/ oder der Jugendlichen erhoben. Bereits 2012 hat ein Pädiater dieses Zertifikat erfolgreich abgeschlossen.

Das neue Spezialarztzertifikat bemisst die Praxisqualität natürlich aus einer Metaperspektive und greift nicht die fachspezifische klinische Qualität auf. Das Projektteam wird trotzdem Pilotzertifikationen mit Exponenten von Fachgesellschaften durchführen und zählt auf eine fruchtbare Zusammenarbeit. Für eine allfällige Weiterentwicklung und Vertiefung des Facharztlabels soll selbstverständlich mit den Fachgesellschaften zusammengearbeitet werden.

\section{Diversifikation in die Romandie}

Die Ausdehnung der Dienstleistungen in die Westschweiz und damit die Bereitstellung der Indikatoren und Standards in französischer Sprache stellen schon länger strategische Prioritäten dar. Bereits kürzerfristig sollen Stiftungsrat und Geschäftsstelle zweisprachig besetzt sein. Die bereits erfolgte Wahl eines Stiftungsrats ${ }^{4}$ aus der Romandie ist in diesem Kontext zu sehen.

Die Auswertung der neuen französischen Version von Modul A erfolgt auf gleiche Art und Weise wie die der deutschen Version über «visotool», die Software von AQUA. Ärztinnen und Ärzte aus der Romandie haben für diese neue Version bereits ihr Interesse angemeldet.

\section{Perspektiven}

Die fundamentale Umstrukturierung der Stiftung in den Jahren 2010 bis 2012 ist erfolgreich abgeschlossen worden und hat neue Perspektiven eröffnet, unter anderem die vorgestellten Neuerungen und Erweiterungen (zusammengefasst in Tabelle 3).
Tabelle 3

Neuentwicklungen EQUAM 2012/13

Innovationen 2012/13

Separates Angebot der Zertifikate zur Ergebnisund Indikationsqualität Module C

(bisher nur als Zusatz-Modul nach bestandenem Basis-Modul EPA)

Zertifikate für Spezialärzte und Pädiater

(Ergänzung von Modul A nach EPA; in Kooperation mit AQUA)

Vorbereitung einer Ausdehnung in die Romandie (Stiftungsrat und Geschäftsstelle zweisprachig, geplante französische Übersetzung der Zertifizierungsunterlagen)

Eines der Schwergewichte der künftigen Entwicklung wird die Erarbeitung neuer klinischer Module $\mathrm{C}$ sein; dasjenige zur Medikationssicherheit ist bereits in Vorbereitung. Diskutiert wird ferner ein Indikatorensatz für Medizinische Versorgungszentren und längerfristig auch für Spitalambulatorien. Ein anderer Akzent liegt auf der mittelfristigen Einführung einer Online-Plattform zur effizienteren Abwicklung der Zertifizierung. Im Weiteren wird sich die Stiftung wie schon bisher - in der Frage der Honorierung von nachgewiesener Qualität engagieren.

All diese Vorhaben sollte und möchte EQUAM nicht allein umsetzen. Es ist seit je ein Credo der Stiftung, dass Fortschritte durch Bündelung der Kräfte erzielt werden sollen. Dies manifestiert sich in zahlreichen Kooperationsverträgen wie auch in der kürzlich lancierten Q-Initiative (SÄZ 10/2013). Doch manche der Akteure hüten peinlich ihre Gärtlein und lassen teils nicht mal einen Einblick zu. Solche Berührungsängste führen dazu, dass das Rad allenthalben immer wieder neu erfunden wird - zweifellos ein unökonomisches Unterfangen und der Sache nicht dienlich.

Mit ihrer Arbeit will die Stiftung nicht mehr und nicht weniger erreichen, als der Ärzteschaft den Weg zur Umsetzung der KVG-Qualitätsanforderungen zu ebnen. Dieses Ziel soll und kann nicht im Alleingang realisiert werden, nicht durch Erfüllung einiger weniger Struktur- und Prozesspostulate und auch nicht durch blosses Selbstdeklarieren der eigenen Praxisqualität.
4 Olivier Girardin, Partner und Geschäftsführer von Hpartner.

AQUA-Institut für angewandte Qualitätsforschung, öttingen, D. 